\title{
CONTROLE DE CADEIRA DE RODAS MOTORIZADA POR MEIO DE COMANDOS MUSCULARES FACIAIS VIA TABLET
}

\author{
C. M. Alves*, L. R. Borges* e E. L. M. Naves* \\ *Universidade Federal de Uberlândia, Uberlândia, Brasil \\ alves.camille@hotmail.com
}

\begin{abstract}
Resumo: Este trabalho apresenta o desenvolvimento de uma ferramenta de controle de cadeira de rodas motorizada por meio de sinais eletromiográficos provenientes do músculo facial masseter. A eletromiografia consiste na análise da atividade muscular, e possui inúmeras aplicações, incluindo estudos na área de Tecnologia Assistiva (TA). O avanço tecnológico nessa área tem permitido uma maior independência para pessoas com deficiência, maior qualidade de vida e inclusão social. Desse modo, é possível desenvolver com o auxilio de ferramentas de eletromiografia, dispositivos para facilitar o controle de cadeiras de rodas motorizadas, visando atender pessoas com deficiências motoras severas. O sistema é composto por um sensor de eletromiografia, Myoware ${ }^{\circledR}$, arduino ${ }^{\circledR}$ para análise do sinal adquirido pelo sensor, um tablet com sistema operacional Android, para interfacear com o usuário, e uma cadeira de rodas adaptada.

Palavras-chave: Tecnologias assistivas, eletromiografia, cadeira de rodas, Android.
\end{abstract}

\begin{abstract}
This paper introduces the development of an application to control a wheelchair through electromyographic signals from the masseter muscle. Electromyography is the analysis of muscle activity, and has numerous applications, including studies in Assistive Technology (AT). A technological advance in this area has allowed greater independence, higher quality of life and social inclusion for people with disabilities. Thus, it is possible to implement electromyography devices to facilitate the control of motorized wheelchairs, for people with more severe disabilities. The system consists of an electromyography sensor Myoware, an Arduino for analysis of the signal acquired by the sensor, a tablet, to display an interface to show the activities for the user and an adapted wheelchair.
\end{abstract}

Keywords: Assistive Technology, eletromyography, wheelchair, Android.

\section{Introdução}

Existem diversos tipos de necessidades especiais, e muitas vezes, o indivíduo que possui alguma limitação se torna dependente de outra pessoa para realizar ações corriqueiras, como se locomover. Atualmente, as cadeiras de rodas motorizadas (CRM) são muito utilizadas, o que facilita bastante na locomoção, no entanto a maioria é controlada por botões ou joysticks, o que impossibilita que algumas pessoas com deficiência motora consigam utilizá-las [1]. Outros meios de controle têm sido desenvolvidos, afim de, atender um maior número de pessoas, como controle por voz, músculos faciais e sinais neurológicos [2].

De acordo com [3] Tecnologia Assistiva engloba todos os dispositivos e soluções que auxiliam pessoas com deficiência física, mental ou emocional. Para [4] a TA pode ser deduzida como um meio que possibilita o aumento da habilidade deficitária, permitindo a realização de funções almejadas e melhorias no campo da comunicação, locomoção, trabalho, aprendizado e qualidade de vida.

A utilização da resposta muscular é uma alternativa extremamente importante, na área de Tecnologia Assistiva, para a viabilização de equipamentos e meios que exercem funções específicas para pessoas que não possuem meios mecânicos para executá-las, como a pressão das mãos ou o ato de andar. Em grande parte dos casos onde o indivíduo possui uma limitação motora muito grande, ainda é possível perceber atividade muscular nos músculos faciais, e a partir da captação dessa atividade pode-se desenvolver meios de interação com o meio onde ele está inserido.

Dada a importância de reintegração das pessoas com deficiências na sociedade atual, aliada ao avanço das tecnologias assistivas. Este trabalho tem como objetivo possibilitar a automação de recursos motores a indivíduos cuja capacidade de locomoção foi reduzida, essa capacidade pode ser restrita apenas a movimentos dos músculos da face. Foi desenvolvido um software para tablets na plataforma Android, que analisa e interpreta sinais eletromiográficos adquiridos através de eletrodos não invasivos posicionados nos músculos da face.

Os sinais adquiridos são utilizados para possibilitar o controle de uma cadeira de rodas possível de ser manuseada por um indivíduo que não deseje ou não possa mais usar seus membros para acioná-la.

\section{Materiais e métodos}

Visando atender as pessoas que possuem um grau mais elevado de dependência foi escolhido o músculo 
masseter, presente na face, para controle da cadeira de rodas. Os principais componentes do sistema incluem sinais EMG captados por eletrodos não invasivos colocados sobre a pele na bochecha do usuário, mais especificamente sobre o músculo masseter, os sinais são coletados por um sensor denominado Myoware, suas informações são processadas por um firmware desenvolvido no Arduino que detecta quando é realizado uma contração e envia para a interface por meio de Bluetooth.

A interface gráfica alterna o escaneamento das opções de controle CRM. Quando recebe uma contração seleciona a opção em destaque e envia o comando para CRM. O diagrama de blocos do sistema completo é mostrado na Figura 1.

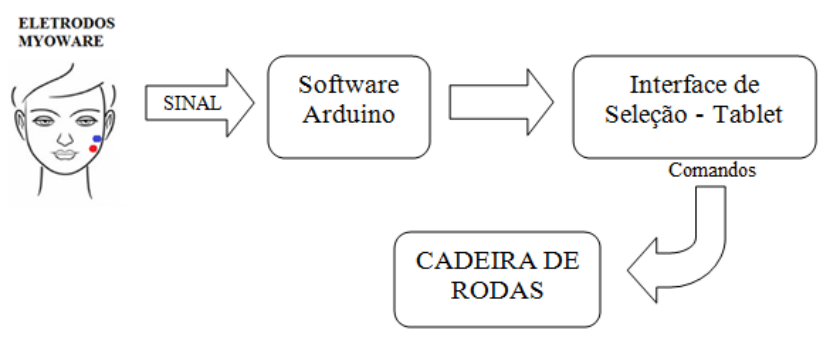

Figura 1: Diagrama de blocos do projeto.

Sinal EMG - Os eletrodos utilizados nesse projeto foram eletrodos superficiais descartáveis. O sensor utilizado para captura dos sinais provenientes dos eletrodos foi o Myoware, que se trata de um sensor que mede a atividade elétrica de um músculo. É projetado para ser utilizado diretamente com um microcontrolador, fornecendo um sinal amplificado, retificado e filtrado que funciona bem com o conversor analógico digital do microcontrolador. $\mathrm{O}$ sensor transmite os dados direto para o Arduino, que é responsável pela digitalização, análise e processamento dos sinais adquiridos. A figura 2 mostra o sensor myoware.

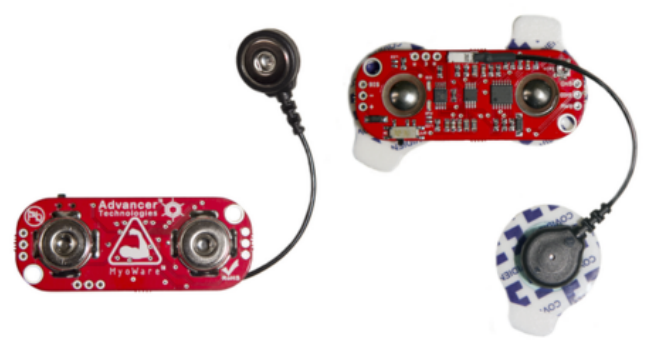

Figura 2: Sensor Myoware.

Para controle da cadeira de rodas utilizou-se o músculo facial masseter. $\mathrm{O}$ mesmo ficou responsável pelos movimentos de direcionar a cadeira para direita ou para esquerda, andar para frente, para trás e parar. A Figura 3 mostra o posicionamento dos eletrodos.

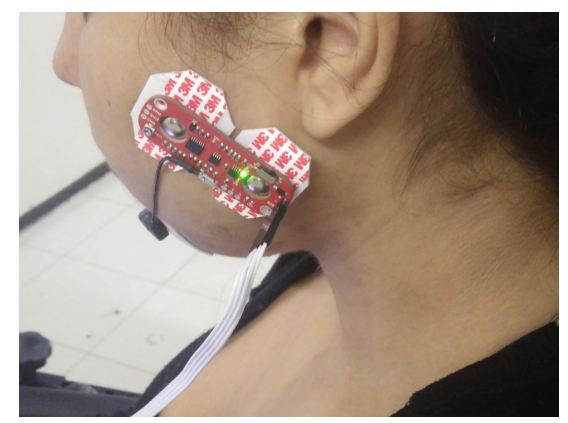

Figura 3: Posicionamento do sensor e dos eletrodos.

Cadeira de rodas - A cadeira de rodas motorizada utilizada no desenvolvimento do projeto foi da marca Seat Mobile do Brasil modelo SM2. Contém duas baterias seladas de $12 \mathrm{~V}$ e $45 \mathrm{Ah}$ cada, e admite velocidade de no máximo $8 \mathrm{~km} / \mathrm{h}$. A Figura 4 mostra a cadeira de rodas adaptada utilizada no projeto.

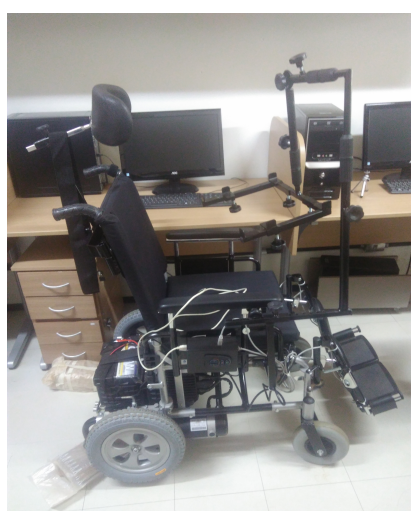

Figura 4: Cadeira de rodas adaptada.

Originalmente os controles da cadeira eram ativados a partir de joystick e botões, no entanto ela foi adaptada para receber diversas formas de controle, como os sinais EMG e EOG. Assim, é necessário realizar o processamento do sinal desejado anteriormente, e indicar qual direção se pretende que o circuito adaptado converte em movimento nos motores.

Software - Para desenvolver o software de seleção dos comandos da cadeira, foi utilizado a plataforma Android Studio, que é um ambiente de desenvolvimento integrado (IDE) elaborado para desenvolver aplicações na plataforma Android. A linguagem de programação é baseada em java e o layout do aplicativo em .xml. O que permite que sejam criados aplicativos para diversos tipos de dispositivos Android, como tablets e smartphones. O Android Studio permite a comunicação de dispositivos Android com o Arduino, por meio de bluetooth ou cabo usb.

O tablet utilizado foi o modelo Galaxy Tab S2 da marca Samsung ${ }^{\text {TM}}$. O sistema operacional é Android. Foi escolhido o sistema Android pois é utilizado por cerca de $80 \%$ da população mundial, com mais de um bilhão de usuários ativos e com mais de um milhão de aplicativos disponíveis [5]. 


\section{Resultados}

Na primeira etapa do projeto, o sensor foi colocado sobre a pele, e o sinal EMG foi enviado para o arduino.

A análise dos dados coletados foi feita no próprio arduino, a pessoa deveria ficar inicialmente em repouso para serem coletados os primeiros dois segundos, de modo que, os dados coletados nesses primeiros segundos são utilizados para cálculo do limiar de contração, é realizado o cálculo do desvio padrão (DP) das amostras desse período. O limiar foi definido como sendo a média do sinal coletado +2.DP. Assim toda vez que o sinal ultrapassasse esse limiar uma contração era detectada. Quando o é realizada uma contração é enviado um comando para o tablet via bluetooth, enquanto não houver contração nenhum sinal é enviado pelo arduino.

A segunda parte do projeto consistiu na criação do aplicativo na plataforma Android. O aplicativo possui cinco opções de comando: frente, ré, esquerda, direita e parar. A interface é composta por cinco botões com imagem, onde é possível ver setas direcionais indicando para qual direção o usuário deseja se movimentar e outros dois botões para iniciar e parar o aplicativo. $\mathrm{Na}$ figura 5 é possível visualizar a interface final no tablet.

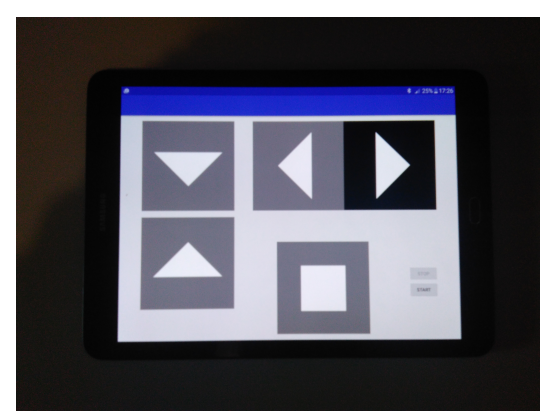

Figura 5: Interface de seleção dos movimentos.

Ao iniciar o aplicativo se conecta automaticamente com o módulo bluetooth para receber os dados do sensor EMG. A comunicação do tablet com a cadeira é feita por meio de cabo USB.

A interface de seleção dos movimentos da cadeira consiste, primeiramente, na varredura automática dos botões frente, ré, esquerda e direita (Figura 6), onde cada botão aparece habilitado para seleção durante um segundo e meio, e ficam alternando até um deles ser selecionado. O botão habilitado para a seleção aparece na cor preta, enquanto os outros permanecem na cor cinza.

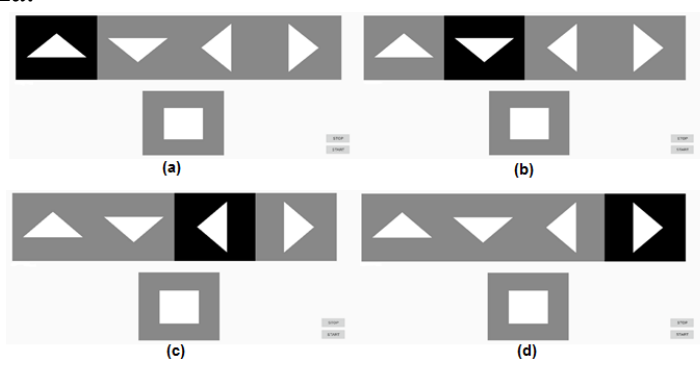

Figura 6: Layout de seleção dos comandos (a) Frente, (b) Trás, (c) Esquerda, (d) Direita.
Quando o usuário desejar acionar um movimento ele contrai o músculo masseter no momento em que a opção está selecionada, ao confirmar o movimento desejado a seta muda para a cor rosa, acionando o movimento da cadeira (Figura 7). É enviado para cadeira um comando de acordo com o movimento ("1" Frente, "3" Direita, "4" Esquerda e "5" Ré).

Ao ser confirmada a seleção de qualquer tipo de movimento da cadeira, automaticamente o único botão possível de ser selecionado é o parar. A pessoa deve realizar outra contração quando decidir parar a cadeira, confirmando a seleção do botão, assim como é feita para os outros comandos. Assim que a pessoa seleciona o botão parar, é enviado o comando "2" para a cadeira por meio de cabo USB. O botão aparece selecionado e novamente se inicia o layout de seleção de um novo comando de locomoção da cadeira.

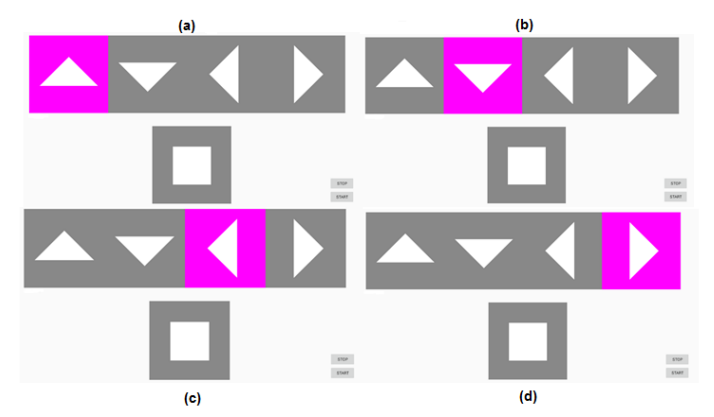

Figura 7: Layout de escolha do comando. (a) Frente, (b) Ré, (c) Esquerda, (d) Direita.

\section{Discussão}

Os resultados finais obtidos do funcionamento do aplicativo na cadeira necessitam de alguns ajustes para operar com maior eficácia, uma vez que, apresentaram alguns problemas na análise dos sinais EMG no processamento realizado na plataforma do arduino. Outra dificuldade encontrada está na escolha do músculo, pois na realização dos testes alguns usuários se queixaram de cansaço muscular devido ao esforço realizado pelo músculo. Este problema poderia ser amenizado se houvesse mais de um grupo muscular para realizar a seleção dos comandos. O protocolo utilizado para seleção também pode dificultar em algumas situações, por exemplo, se o usuário quiser realizar dois movimentos diferentes de forma rápida ele deverá esperar pelo menos um segundo e meio entre a primeira a ação e a de parada para poder selecionar uma segunda opção de controle.

\section{Conclusão}

O desenvolvimento de novos meios que permitam o controle de cadeiras de rodas acessível para muitos usuários, que necessitam de alternativas diferentes do usual, é uma área em expansão. A fim de atender o maior número possível de usuários a interface deve ser 
composta por opções diversas de acionamento para um comando, pois cada usuário possui necessidades diferentes.

A tecnologia assistiva é uma ferramenta extremamente importante no estudo e desenvolvimento de dispositivos capazes de reintegrar funções para pessoas com deficiências.

O objetivo deste trabalho foi desenvolver um aplicativo para sistemas Android que fosse capaz de controlar uma cadeira de rodas adaptada por meio de sinais eletromiográficos, obtidos através da contração do músculo facial masseter. $\mathrm{O}$ firmware de aquisição e análise dos dados foi desenvolvido no Arduino e o software com a interface foi desenvolvido no Android Studio na linguagem Java.

Pode-se perceber que os resultados obtidos durante o desenvolvimento do aplicativo foram bastante satisfatórios, apesar das dificuldades encontradas, o sistema operou conforme o esperado. A partir dos resultados obtidos, é possível concluir que um usuário com algum tipo de deficiência severa, na qual o comprometimento motor é alto, pode se tornar independente para realizar atividades básicas como se locomover, resultando em uma maior qualidade de vida.

\section{Agradecimentos}

Os autores agradecem ao CNPq, à CAPES e a FAPEMIG pelo apoio financeiro para esta pesquisa.

\section{Referências}

[1] ALBRECHT, B. L. Controle de uma cadeira de rodas motorizada através de eletromiografia em uma plataforma embarcada. Trabalho de conclusão de curso de graduação em Engenharia Elétrica. Universidade Federal do Rio Grande do Sul: Porto Alegre, 2010.

[2] CHAMPATY, B., DUBEY, P., SAHOO, S., RAY, S.S., PAL, K., ANIS, A., Development of wireless EMG control system for rehabilitation devices, in: Proceedings of the Annual International Conference on Emerging Research Areas: Magnetics, Machines and Drivers, 2014.

[3] LAPLANTE, M. P.; HENDERSHOT, G. E.; MOSS, A. J.. Assistive Technology Devices and Home Accessibility: Features: Prevalence, Payment, Need, and Trends. Advance Data From Vital And Health Statistics, Hyattsville, Md., v. 217, p.2-5, 16 set. 1992.

[4] BERSCH, R.. INTRODUÇÃO À TECNOLOGIA ASSISTIVA. Porto Alegre - RS: Cedi - Centro Especializado em Desenvolvimento Infantil, 2008.

[5]Dados do sistema operacional móvel Android, d i s p o níve 1 $<\underline{\text { http://www.tecmundo.com.br/sistema- }}$ operacional/60596-ios-android-windows-phonenumeros-gigantes-comparados-infografico.htm $>$. Acesso em: 12/06/2016. 